\title{
Realtime Luminance Analyse
}

\begin{abstract}
This paper presents the progress in the development of luminance analyzer LumiDISP. The main innovation is the processing of luminance analysis in real time by using industrial cameras. This new feature allows more efficient process of measurement, because the system allows interactive work. The paper describes the main differences of working with previously used digital camera and demonstrates measurement UGR using the new methodology.
\end{abstract}

Keywords: luminance analysis, industrial camera, real-time processing

\section{Introduction}

Our team is several years working on development of the system for luminance analysis using digital photography called LumiDISP [1]. To capture a scene has been yet used professional digital camera that was precisely calibrated for the measurement. We typically use digital SLR cameras Nikon. There are various conditions in which the system is used in practice. Different requirements are imposed on the measurement of day light and measurement e.g. of lighting or visibility of pedestrians on the road during the night. Basic features of digital camera that affect the acquired data is shutter time, aperture and sensitivity. All these parameters we can precisely calibrate for each piece of the camera so that the camera can be used for different types of measurements with different conditions. If the dynamic range of the camera sensor is not enough for the scene, the scene may be photographed several times with different parameters, and then can be created so-called HDR image. The entire measurement process is usually divided into two phases, images of scene are captured in the first phase and the subsequent phase is data analysis. Processing of single image takes typically half a minute excluding duration of capturing, depending on the computer's performance. This time includes transfer from camera to PC via USB, image decoding and conversion of RGB color values to the luminance values according to the appropriate calibration function. Measured values cannot be directly read during capturing because of this delay, operator must wait for data processing, or perform additional analysis later. But this prevents any interaction with the measured object, if it is needed at the base of the resulting data. So far, this procedure was acceptable but we have already encountered situations, when it would be preferable to have the measured values in real time.

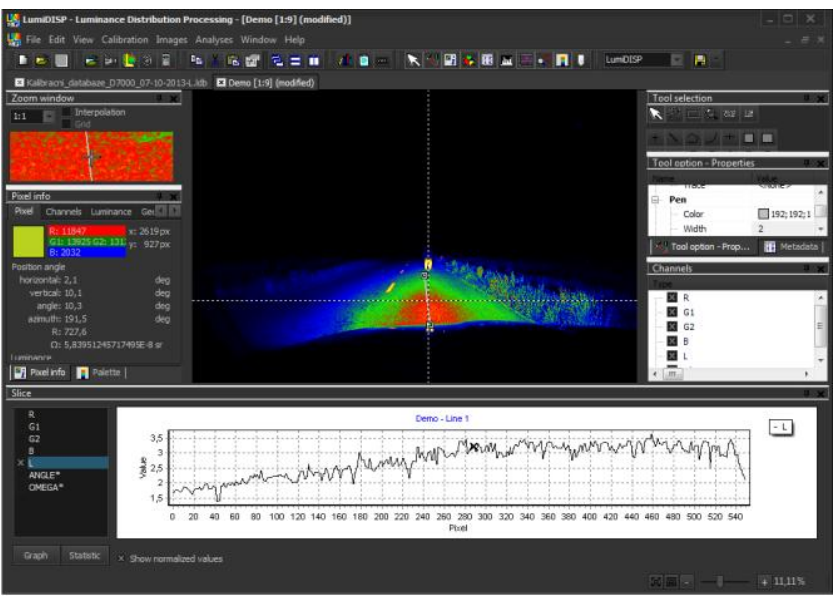

Fig.1. Software LumiDISP
To eliminate this problem it is important to find a suitable sensor that will meet the requirements for luminance analysis. We decided to use an industrial video camera, which is able to provide the raw data from the sensor at 25 or more frames per second. The desired result is to obtain immediate response of the measuring system, ie. system will interactively display the measured values at selected points of the scene. Using the industrial camera brings other problems that need to be solved. Selected aspects are discussed in the following sections.

\section{Used devices}

When selecting a suitable camera it is necessary to respect the conditions which must be met with regard to the further processing of the captured data. The basic requirement is that the camera must provide raw data from the sensor without any modification, what allows its accurate calibration. Conventional cameras used for image recording typically perform various color corrections and then save images using a lossy compression, so the original data is always greatly distorted. This requirement satisfies industrial cameras, which are used for machine vision. For our experiments was chosen camera from the renowned manufacturer Basler Ace acA2000-50gc [2]. Selected parameters of this camera are shown in Table 1.

Table 1. Parameters of the camera

\begin{tabular}{|l|l|}
\hline Resolution & $2046 \mathrm{px} \times 1086 \mathrm{px}$ \\
\hline Pixel Size & $5.5 \mu \mathrm{m} \times 5.5 \mu \mathrm{m}$ \\
\hline Frame Rate & $50 \mathrm{fps}$ \\
\hline Video Output & Mono 8, Bayer GR 8, Bayer GR 12, Bayer \\
Format & GR 12 Packed, YUV 4:2:2 Packed, YUV \\
& $4: 2: 2$ (YUYV) Packed \\
\hline Pixel Bit Depth & 12 bits \\
\hline Exposure Control & $\begin{array}{l}\text {-programmable via the camera API } \\
\text {-external trigger signal }\end{array}$ \\
\hline Sensor Type & CMOS 2/3" \\
\hline Sensor Size & $11.26 \mathrm{~mm} \times 5.98 \mathrm{~mm}$ \\
\hline Sensitivity & 5,56 V/lux.S \\
\hline Dynamic range & $60 \mathrm{~dB}$ \\
\hline
\end{tabular}

Camera meets the basic requirement, it provides the raw data from the sensor chip. The camera can shoot the scene at speeds up to 50 frames per second. Also important is the possibility to control exposure time by software, which will make possible to measure luminance in various ranges. Resolution of industrial camera is $2 \mathrm{Mpx}$, which is compared to previously used Nikon cameras with $16 \mathrm{Mpx}$ much less. However regarding the amount of data processed, the work with this camera is more complicated, since the images are processed in real time several times per second. Due to the large amount of data for transmission from the camera is used Gigabit Ethernet. 
During the measurement the smaller resolution is compensated by interactive respond of the system. If SLR camera is used usually whole scene is captured at high resolution and subsequently is performed analysis of selected parts of the scene. When using the camera working in real time, the operator can select the desired parts of the scene and evaluate them immediately.

Table 2. Parameters of the lens

\begin{tabular}{|l|l|}
\hline Focal length & $2.7 \mathrm{~mm}$ \\
\hline Iris Range & F1.8-16 \\
\hline Focus & Fixed \\
\hline Angular Field of View & $\begin{array}{l}185^{\circ} \times 185^{\circ}(1 "-8.6 \mathrm{~mm} \text { diag.) } \\
185^{\circ} \times 140^{\circ}\left(2 / 3^{\prime \prime}\right) \\
\\
136^{\circ} \times 102^{\circ}\left(1 / 2^{\prime \prime}\right)\end{array}$ \\
\hline Resolution & $5 \mathrm{Mpx}$ \\
\hline Mount & $\mathrm{C}$ \\
\hline
\end{tabular}

Together with the camera is used to capture images fisheye lens, namely model Fujifilm FE185C086HA-1 [3] The selected camera lets you mount any lens with a standard C-mount so the lens may be replaced by a specific type according to the measurement. Basic parameters of the lens are shown in Table 2. The lens has a fixed focal length and manually adjustable aperture. Selected camera and lens show Figure 2.
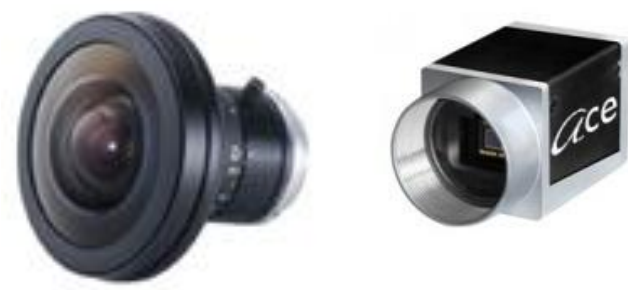

Fig.2. Used camera and lens

Using described devices can be captured full-motion video sequences of measured scenes with a wide viewing angle about 180 degrees. An example of the obtained data shows Figure 3.

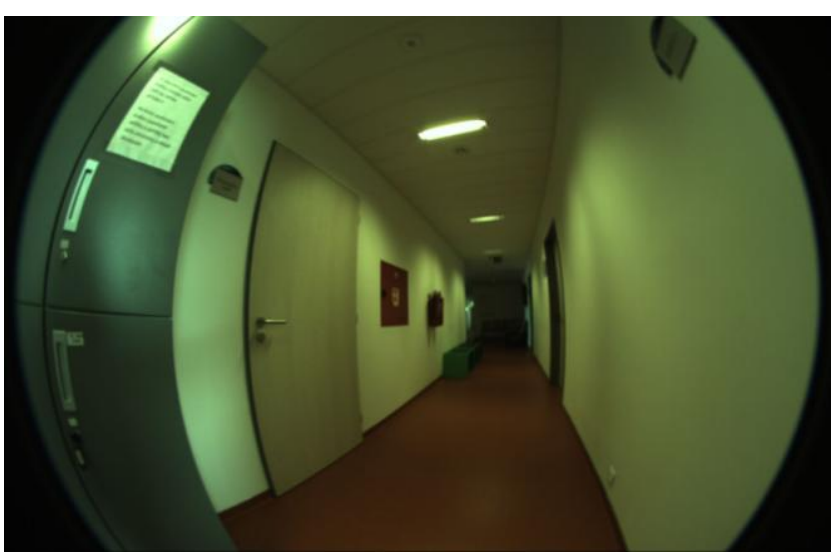

Fig.3. Example of source image from camera

\section{Principle of processing}

Processing of images from industrial cameras is carried out in a similar way as using a professional SLR camera. From the individual color components $R, G$, B, which are distributed on a sensor in so called Bayer mask, at first is determined the full RGB image. Subsequently is using the calibration function of the camera sensor computed the luminance value of each pixel.

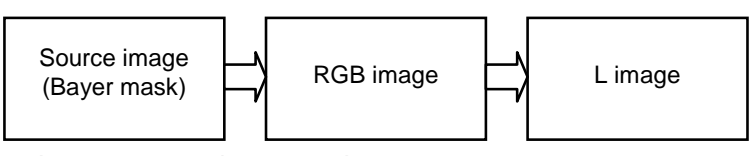

Fig.4. Image processing procedure

Calibration of the sensor is based on the sensitivity of the sensor for each color component. Figure 3 shows a graph of the sensitivity of the sensor provided by the manufacturer of device, however for accurate measurement it is always necessary to calibrate a particular camera.

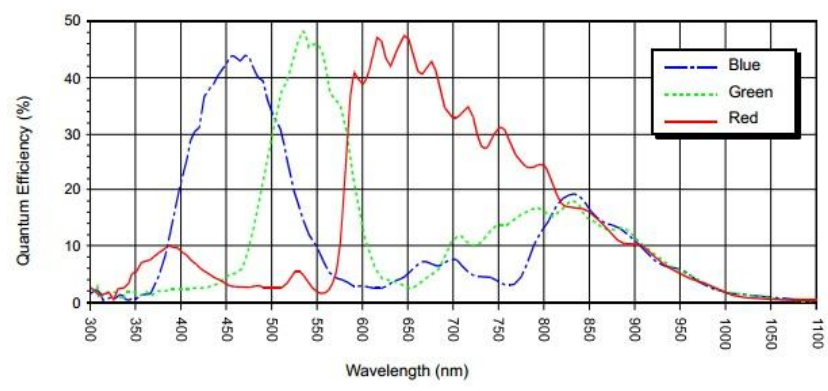

Fig.5. Sensitivity of the sensor

Besides information about luminance the geometry data of evaluated scene may be necessary for some measurements too. The spatial angle of the selected area is used, the angle of the selected pixel and so on. These data are calculated on the basis of calibration of the lens, which can also be carried out before own measurement. Subsequently, after determining luminance and image geometry analysis can be performed on selected areas of the image using so called detectors and other tools available in the program. More detailed description of the calibration and functions of program can be found in our previous paper [4].

Even if the general principle of calculation remains unchanged, time requirements for the implementation of individual operations significantly differ. While for the conventional SLR camera, images are processed later and calculation of a single frame can take several seconds depending on the hardware, the data from industrial camera have to be processed in real time, ie. a few frames per second. Therefore, it was necessary to implement a series of optimizations that enable the processing of data from the camera in real time. Raw data stream from the camera is in the order of $100 \mathrm{MB} / \mathrm{s}$. Mlti-core processors are fully utilized, some calculations are additionally accelerated using the graphics card. The fundamental difference when shooting with the industrial camera to a SLR camera is the way of realization of shutter. The mechanical plane shutter is usually in SLR cameras, which for the selected time period exposes scanning sensor. Each such camera has defined guaranteed number of shutter clicks. The industrial camera has electronically realized shutter, there is controlled time during the sensor receives photons. For selected cameras this period may be adjusted from microsonds to a few seconds. The shortest time is used when scanning big intensities eg. daylight, a longer period shall be used when measuring lower values of the luminance. If the shutter time will be longer than the frame interval, frame rate will be reduced, but still the measurements will be performed interactively. Resolution of the sensor is 12 bits, it also define it's the dynamic range. If the measured scene contains luminance with great dynamics, it can be like with SLR camera to created socalled HDR image. Measurements can be repeated several times with different shutter time and then is composed the resulting image with high range. However, the problem can 
cause any movement of the camera during the measurement. Therefore, it is appropriate to add to the algorithm for image stabilization, which enables to align the following images. The basic principle of such algorithms is that the camera motion is estimated in each frame and subsequent images are transformed so that this movement is eliminated. These tasks are solved in machine vision, a detailed description would be beyond the scope of this paper.

\section{Evaluation of UGR in real time}

LumiDISP program already contains a module for calculating the glare using UGR method [5]. This module has been optimized so as to be able to process the data coming from the online camera. On this task it was tested whether it is realistic that the luminance analysis could be done in this manner. Besides optimization of the calculations it was necessary to implement a fully automated detection of light sources in the scene, whose positions are important to evaluate the UGR. During the offline analysis the position of light sources can be set or corrected manually, but in this real time case must be positions found without operator intervention. The calculation is based on the setting of the properties of light sources, such as their color and the estimated luminance. Procedure for processing each image from the camera is following:

Computation of RGB image and luminance image Computation of lens geometry and evaluation of position index

Automatic detection of light sources

Evaluation of UGR

The result of the calculation is both luminance image, which can be seen in the distribution of luminance in different places of the scene, and especially value of glare factor. The advantage compared to the previous measurement method is that the value of the glare factor can be read interactively in any position of the camera, and can be quickly assessed the entire situation in a given area. Currently the program is able to performing measurement of UGR at rate 1 frame per second, which is sufficient for the initial experiments. We expect that after the implementation of further optimizing the calculation speed will increase. Figure 6 show example such light analysis, automatically detected light sources are marked by red color.

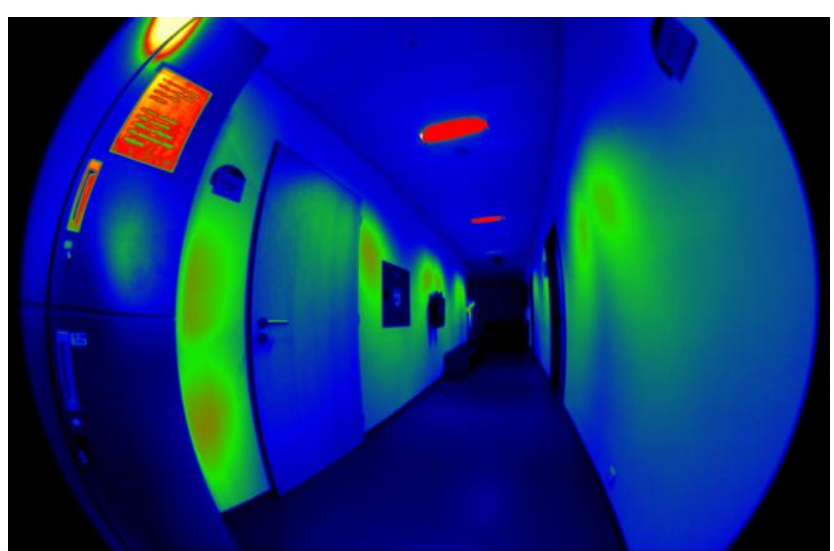

Fig.6. Example of UGR analysis

\section{Conclusion}

The paper introduced new functions of program LumiDISP allowing processing images from industrial cameras. Currently there are basic functions for calculating the luminance image in real time. To demonstrate the enhanced luminance analysis was also carried out an evaluation of UGR. Subsequently it is planned to perform a comparative measurement for verifying the operation of the system under different conditions and compared with the accuracy of converted using previously used digital camera. It remains to solve other differences of processing data between SLR and industrial cameras eg. method of archiving recorded video sequences, which will be difficult due to the volume of obtained data. In the future, we plan to further expand the capabilities luminance analysis in real time. One of the planned features is for example 3D reconstruction of measured scenes from captured video sequences. The output of the program will be the 3D scene is supplemented with information about lighting.

\section{ACKNOWLEDGEMENTS}

This research work has been carried out in the Centre for Research and Utilization of Renewable Energy (CVVOZE). Authors gratefully acknowledge financial support from the Ministry of Education, Youth and Sports of the Czech Republic under NPU I programme (project No. LO1210).

\section{REFERENCES}

[1] LumiDISP: Software for luminance analyse. Available from WWW: http://www.lumidisp.eu.

[2] Basler Ace acA2000-50gc. Available from WWW: http://www.baslerweb.com/en/products/area-scancameras/ace/aca2000-50gc.

[3] Fujifilm FE185C086HA-1. Available from WWW: http://www.fujifilmusa.com/products/optical devices/security/fi sh-eye/5-mp/fe185c086ha-1.

[4] SUMEC, S.; ŠKODA, J.; KRBAL, M.; BAXANT, P. Evaluation of illumination using digital photography. In Proceedings of CIE Centenary Conference "Towards a New Century of Light". Paris: COMMISSION INTERNATIONALE DE L ECLAIRAGE, 2013. s. 1208-1215. ISBN: 978-3-902842-44-2.

[5] CIE 117:1995. Discomfort Glare in Interior Lighting. Vienna, Austria: CIE, 1995. ISBN 978-3-900734-70-1.

\footnotetext{
Authors:

Ing. Stanislav Sumec, Ph.D., Brno University of Technology, Faculty of Electrical Engineering and Communication, Technická 3082/12, 61600 Brno, e-mail: sumec@feec.vutbr.cz,

Ing. Jan Škoda, Ph.D., Brno University of Technology, Faculty of Electrical Engineering and Communication, Technická 3082/12, 61600 Brno, e-mail: skoda@feec.vutbr.cz,

Assoc. Prof. Ing. Petr Baxant, Ph.D., Brno University of Technology, Faculty of Electrical Engineering and Communication, Technická 3082/12, 61600 Brno, e-mail: baxant@feec.vutbr.cz Ing. Michal Krbal, Ph.D., Brno University of Technology, Faculty of Electrical Engineering and Communication, Technická 3082/12, 61600 Brno, e-mail: krbal@feec.vutbr.cz.
} 